\title{
On Some Numbers Related to Extremal Combinatorial Sum Problems
}

\author{
D. Petrassi \\ Dipartimento di Matematica e Informatica, Università della Calabria, Via Pietro Bucci, 87036 Arcavacata di Rende, Italy
}

Correspondence should be addressed to D. Petrassi; petrassidaniele@gmail.com

Received 30 October 2013; Accepted 7 January 2014; Published 3 March 2014

Academic Editor: Franck Petit

Copyright (C) 2014 D. Petrassi. This is an open access article distributed under the Creative Commons Attribution License, which permits unrestricted use, distribution, and reproduction in any medium, provided the original work is properly cited.

Let $n, d$, and $r$ be three integers such that $1 \leq r, d \leq n$. Chiaselotti (2002) defined $\gamma(n, d, r)$ as the minimum number of the nonnegative partial sums with $d$ summands of a sum $\sum_{1=1}^{n} a_{i} \geq 0$, where $a_{1}, \ldots, a_{n}$ are $n$ real numbers arbitrarily chosen in such a way that $r$ of them are nonnegative and the remaining $n-r$ are negative. Chiaselotti (2002) and Chiaselotti et al. (2008) determine the values of $\gamma(n, d, r)$ for particular infinite ranges of the integer parameters $n, d$, and $r$. In this paper we continue their approach on this problem and we prove the following results: (i) $\gamma(n, d, r) \leq\left(\begin{array}{c}r \\ d\end{array}\right)+\left(\begin{array}{c}r \\ d-1\end{array}\right)$ for all values of $n, d$, and $r$ such that $((d-1) / d)(n-1) \leq$ $r \leq((d-1) / d) n$; (ii) $\gamma(d+2, d, d)=d+1$.

\section{Introduction}

Let $n, d$, and $r$ be three fixed integers such that $1 \leq r, d \leq n$. We set $[n]:=\{1, \ldots, n\}$ and

$$
W_{n}:=\left\{f:[n] \longrightarrow \mathbb{R}: \sum_{x \in[n]} f(x) \geq 0\right\} .
$$

The elements of $W_{n}$ are called $n$-weight functions and, if $f \in$ $W_{n}$, we set $f^{+}=|\{a \in[n]: f(a) \geq 0\}|$. For example, if $f(1)=f(2)=f(3)=1, f(4)=f(5)=-1 / 2$, and $f(6)=$ $f(7)=-1$, then $f^{+}=3$. If $f \in W_{n}$, we also set $\Phi(f, d):=$ $\left\{A \subseteq[n]:|A|=d, \sum_{a \in A} f(a) \geq 0\right\}$ (we call $\left(d^{+}, n\right)$-subset of $f$ a generic element of $\Phi(f, d)), \phi(f, d):=|\Phi(f, d)|$, and

$$
\gamma(n, d, r):=\min \left\{\phi(f, d): f \in W_{n}, f^{+}=r\right\} .
$$

These numbers $\gamma(n, d, r)$ were introduced in [1] in order to refine the study of a conjecture of Manickam-MiklósSinghi (for further information on this conjecture and on its links with the numbers $\gamma(n, d, r)$ see [1-4]). The complete determination of the numbers $\gamma(n, d, r)$ is a very difficult task and actually they are known only for a relatively small range of the integer parameters $n, d$, and $r$. In [1-3] some of the numbers $\gamma(n, d, r)$ have been determined, and we report these values:

$$
\begin{aligned}
& \left(\begin{array}{l}
n-1 \\
d-1
\end{array}\right) \text { if } r \leq d \leq n / 2, \\
& \left(\begin{array}{l}
n-r \\
d-r
\end{array}\right) \text { if } r \leq d<n \text { and } r<(n /(n-d)), \\
& \left(\begin{array}{l}
r \\
d
\end{array}\right) \text { if } d<r<n \text { and } r>((d-1) / d) n, \\
& \left(\begin{array}{l}
n-1 \\
d-1
\end{array}\right) \text { if } r=1, \\
& \left(\begin{array}{l}
r \\
d
\end{array}\right)+\left(\begin{array}{l}
r-1 \\
d-1
\end{array}\right) \text { if } r \geq d \text { and } r=((d-1) / d) n, \\
& \left(\begin{array}{l}
r \\
d
\end{array}\right)+\left(\begin{array}{l}
r-1 \\
d-1
\end{array}\right) \text { if } n=2 d+2, r=2 d-1 \text { and } d \geq 2 .
\end{aligned}
$$

In particular, in [3] the authors prove the last of these results using Hall's matching theorem.

Also, in $[2,5]$ the numbers $\gamma(n, d, r)$ were linked within the context of the combinatorial order theory. More in detail, in [2] the authors introduce two new classes of lattices of signed integer partitions, $S(n, r)$ and $S(n, d, r)$, and they show that the numbers $\gamma(n, d, r)$ can be interpreted as the cardinality of particular types of up-sets in the previous lattices.

On the other hand, the lattices $S(n, r)$ and $S(n, d, r)$ can also be considered as particular types of discrete dynamical systems. In this context many properties of the numbers $\gamma(n, d, r)$ can be related to the evolution rules that characterize $S(n, r)$ and $S(n, d, r)$ as discrete dynamical systems (see $[6,7])$. For very recent studies concerning the discrete dynamical systems see [8-12].

In this paper we determine some new identities and new bounds for the numbers $\gamma(n, d, r)$. In particular, we show that 
(i) $\gamma(n, d, r) \leq\left(\begin{array}{c}r \\ d\end{array}\right)+\left(\begin{array}{c}r \\ d-1\end{array}\right)$ for all values of $n, d$, and $r$ satisfying $((d-1) / d)(n-1) \leq r \leq((d-1) / d) n$ (Corollary 5),

(ii) $\gamma(n, d, r)=\left(\begin{array}{c}r \\ d\end{array}\right)+\left(\begin{array}{c}r \\ d-1\end{array}\right)=d+1$ in the case $n=d+2$ and $r=d$ (Proposition 8).

Finally we provide a combinatorial interpretation of the inequality $((d-1) / d)(n-1)<r \leq((d-1) / d) n$.

The remaining part of this paper is structured as follows. In Section 2 we provide the necessary notations for the sequel. In Section 3 we establish our results and, finally, in Section 4 we briefly describe conclusions and possible future research approaches.

\section{Notations}

In the sequel, we will assume that a generic weight function $f \in W_{n}$, with $f^{+}=r$, has the form

$$
\begin{array}{cccccc}
1 & \cdots & r & r+1 & \cdots & n \\
\downarrow & \cdots & \downarrow & \downarrow & \cdots & \downarrow \\
x_{1} & \cdots & x_{r} & y_{1} & \cdots & y_{n-r},
\end{array}
$$

with

$$
x_{1} \geq x_{2} \geq \cdots \geq x_{r} \geq 0>y_{1} \geq y_{2} \geq \cdots \geq y_{n-r} .
$$

Let us call the indexes $1, \ldots, r$ the nonnegative elements of $f$ and the indexes $r+1, \ldots, n$ the negative elements of $f$. The real numbers $x_{1}, \ldots, x_{r}$ are said to be the nonnegative values of $f$ and the numbers $y_{1}, \ldots, y_{n-r}$ are said to be the negative values of $f$.

If $i_{1}, \ldots, i_{\alpha}$ are nonnegative elements of $f$ and $j_{1}, \ldots, j_{\beta}$ are negative elements of $f$, with $i_{1}<\cdots<i_{\alpha}$ and $j_{1}<\cdots<j_{\beta}$, a subset $A$ of $\{1, \ldots, n\}$ is said to be of type

$$
\left[i_{1}, \ldots, i_{\alpha}\right]_{a}^{+}\left[j_{1}, \ldots, j_{\beta}\right]_{b}^{-}
$$

if $A$ is made of $a$ elements chosen in $\left\{i_{1}, \ldots, i_{\alpha}\right\}$ and $b$ elements chosen in $\left\{j_{1}, \ldots, j_{\beta}\right\}$.

Let $X$ be a finite set of integers. If $q$ is an integer less than or equal to $|X|$, we call $q$-string on $X$ a sequence $a_{1}, \ldots, a_{q}$, where $a_{1}, \ldots, a_{q}$ are distinct elements of $X$ such that $a_{1}<$ $\cdots<a_{q}$. In this paper, each subset $Y$ of $X$ with $q$ elements will be identified with the $q$-string of its elements ordered in increasing way. When $i_{1}, \ldots, i_{k}$ are nonnegative elements of $f$ and $j_{1}, \ldots, j_{l}$ are negative elements of $f$, with $i_{1}<\cdots<i_{k}<$ $j_{1}<\cdots<j_{l}$, the $(k+l)$-string $i_{1} \cdots i_{k} j_{1} \cdots j_{l}$ will be written in the form

$$
i_{1} \cdots i_{k} \mid\left(j_{1}-r\right) \cdots\left(j_{l}-r\right)
$$

(thus $j_{1}-r, \ldots, j_{l}-r \in\{1, \ldots, n-r\}$ ).

For example, if $n=10$ and $r=7$, the 4-string 1269 will be written in the form $126 \mid 2$.

Using the string-terminology instead of the setterminology, in the sequel, we will call a $\left(d^{+}, n\right)$-subset of $f$ a $\left(d^{+}, n\right)$-string of $f$.

\section{The Results}

The range of values of the integer parameters $n, d$, and $r$ that we are going to study is the following: $d \leq r \leq n$. As reported in the first section, we already know that the next result holds for $\gamma(n, d, r)$ in the case $r>((d-1) / d) n$. We state it referring to its proof.

Proposition 1. If $r \geq d$ and $r>((d-1) / d) n$, then $\gamma(n, d, r)=$ $\left(\begin{array}{l}r \\ d\end{array}\right)$.

Proof. See [1].

Therefore we concentrate our attention on the case $r \leq$ $((d-1) / d) n$. In order to examine it, we start by considering the partition $\mathscr{P}$ of the real interval $(0,((d-1) / d) n]$ :

$$
\mathscr{P}=\left\{0, \frac{d-1}{d}, 2 \frac{d-1}{d}, \ldots,(n-1) \frac{d-1}{d}, n \frac{d-1}{d}\right\} .
$$

The following proposition establishes when an interval determined by $\mathscr{P}$ contains an integer.

Proposition 2. If $k=1, \ldots, n$ and if $n-k \neq_{d} 0$, there exists a unique integer $r$ such that

$$
\frac{d-1}{d}(n-k)<r \leq \frac{d-1}{d}(n-k+1),
$$

and $r$ coincides with $\lfloor((d-1) / d)(n-k+1)\rfloor$. Furthermore if $n-k \equiv{ }_{d} 0$, no integer $r$ satisfies (8).

Proof. Let $k \in\{1, \ldots, n\}$ and set $m=n-k+1$. Since the interval $(((d-1) / d)(m-1),((d-1) / d) m]$ has length $((d-$ $1) / d)<1$, there is at most one integer $r$ that satisfies (8). Let us now write $m$ in the form

$$
m=\tilde{q} d+s,
$$

where $\tilde{q}, s$ are integers such that $\tilde{q} \geq 0,1 \leq s \leq d$. Let us suppose now that $n-k \not_{d} 0$; that is, $m \neq_{d} 1$; then we have $2 \leq$ $s \leq d$.

Let $r=\lfloor((d-1) / d) m\rfloor$. We show that $r$ satisfies $(8)$.

Firstly, the second inequality is straightforward; secondly, for the first inequality we observe

$$
\begin{aligned}
\frac{d-1}{d}(m-1) & =\frac{d-1}{d}(\widetilde{q} d+s-1) \\
& =\widetilde{q}(d-1)+(s-1) \frac{d-1}{d} .
\end{aligned}
$$

Furthermore

$$
\begin{aligned}
r=\left\lfloor\frac{d-1}{d}(\tilde{q} d+s)\right\rfloor & =\left\lfloor\tilde{q}(d-1)+s-\frac{s}{d}\right\rfloor \\
& =\widetilde{q}(d-1)+(s-1) .
\end{aligned}
$$

Therefore $r>((d-1) / d)(m-1)$, since $s \geq 2$.

If $n-k \equiv_{d} 0$, that is, $m \equiv_{d} 1$, in (9), we have $s=1$ and (8) becomes

$$
\tilde{q}(d-1)<r \leq \tilde{q}(d-1)+\frac{d-1}{d} .
$$

Note that (12) has no integer solutions. 
Lemma 3. Let $r$ be a positive integer such that

$$
d \leq r \leq \frac{d-1}{d} n
$$

Then there exists a unique positive integer $b(r) \in\{1, \ldots, n-r-$ 1) that satisfies

$$
\frac{d-1}{d}(n-b(r))<r \leq \frac{d-1}{d}(n-b(r)+1) .
$$

Proof. By construction of partition $\mathscr{P}$, as in (7), there exists a unique $b(r) \in\{1, \ldots, n\}$ such that (14) holds.

We now show that $b(r)$ cannot exceed $n-r-1$.

Firstly, we suppose that $b(r)>n-r$. Then, we write $b(r)$ in the form $b(r)=n-r+\zeta$, with $\zeta$ integer such that $1 \leq \zeta \leq r$. Since $r$ satisfies (14), we have

$$
\frac{d-1}{d}(r-\zeta)<r \leq \frac{d-1}{d}(r-\zeta+1) ;
$$

that is,

$$
\zeta(1-d)<r \leq(d-1)(1-\zeta)
$$

Since $\zeta(1-d)<0$ and $(d-1)(1-\zeta) \leq 0$, there is no positive integer $r$ that satisfies (16).

Secondly, if $b(r)=n-r$, (14) becomes

$$
\frac{d-1}{d} r<r \leq \frac{d-1}{d}(r+1) \text {; }
$$

that is,

$$
0<r \leq d-1
$$

contradicting the hypothesis (13).
Using the previous lemma, we find a useful and appreciable upper bound for $\gamma(n, d, r)$. This is one of our important steps to establish, under suitable hypotheses for $n, d$, and $r$, an exact value for $\gamma(n, d, r)$.

Proposition 4. Let $r$ be a positive integer that satisfies

$$
d \leq r \leq \frac{d-1}{d} n
$$

then

$$
\gamma(n, d, r) \leq \sum_{j=0}^{\min \{b(r), d-1\}}\left(\begin{array}{c}
b(r) \\
j
\end{array}\right)\left(\begin{array}{c}
r \\
d-j
\end{array}\right) .
$$

Proof. Since $1 \leq b(r) \leq n-r-1$, we construct a weight function $f \in W_{n}$, with $f^{+}=r$, such that

$$
\phi(f, d)=\sum_{j=0}^{\min \{b(r), d-1\}}\left(\begin{array}{c}
b(r) \\
j
\end{array}\right)\left(\begin{array}{c}
r \\
d-j
\end{array}\right) .
$$

This is sufficient to prove the (20).

Let $h=\min \{b(r), d-1\}$. Let $\alpha$ be a positive real number. In order to simplify the notation, we call $\beta$ the number $((r+$ $b(r)(-\alpha)) /(n-r-b(r)))$, in such a way that

$$
r+b(r)(-\alpha)+(n-r-b(r))(-\beta)=0
$$

holds.

At this point we define the function

$$
\begin{aligned}
& 1 \cdots r r+1 \cdots r+b(r) r+(b(r)+1) \cdots r+(n-r)
\end{aligned}
$$

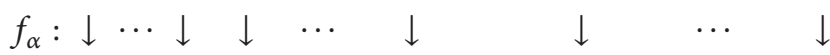

$$
\begin{aligned}
& \begin{array}{llllllllll}
1 & \cdots & 1 & -\alpha & \cdots & -\alpha & & -\beta & \cdots & -\beta .
\end{array}
\end{aligned}
$$

We now show that, for $\alpha$ sufficiently small, that is,

$$
0<\alpha<\min \left\{\frac{r}{b(r)}, \frac{d}{h}-1, \frac{d}{b(r)}\left(r-\frac{d-1}{d}(n-b(r))\right)\right\},
$$

$f_{\alpha}$ is a weight function that satisfies (21). In fact, (a) the denominator of $\beta$, due to Lemma 3, is a positive number. Furthermore the numerator of $\beta$ is a positive number if and only if $\alpha<(r / b(r))$. Therefore (24) and the definition of $\beta$ assure that $f_{\alpha}$ is a weight function.

(b) Having $\alpha<d / h-1$ is equivalent to require

$$
\underbrace{1+\cdots+1}_{d-h \text { times }}+\underbrace{(-\alpha)+\cdots+(-\alpha)}_{h \text { times }}>0 \text {. }
$$

This condition assures that the subsets of the type 


$$
\begin{array}{cccc}
{[1, \ldots, r]_{d}^{+}} & {[r+1, \ldots, r+b(r)]_{0}^{-},} & \text {in total } & \left(\begin{array}{c}
b(r) \\
0
\end{array}\right)\left(\begin{array}{c}
r \\
d
\end{array}\right) \\
{[1, \ldots, r]_{d-1}^{+}} & {[r+1, \ldots, r+b(r)]_{1}^{-},} & \text {in total } & \left(\begin{array}{c}
b(r) \\
1
\end{array}\right)\left(\begin{array}{c}
r \\
d-1
\end{array}\right) \\
\vdots & \vdots & \vdots \\
{[1, \ldots, r]_{d-h}^{+}} & {[r+1, \ldots, r+b(r)]_{h}^{-},} & \text {in total }\left(\begin{array}{c}
b(r) \\
h
\end{array}\right)\left(\begin{array}{c}
r \\
d-h
\end{array}\right)
\end{array}
$$

are $\left(d^{+}, n\right)$-subsets of $f_{\alpha}$.

(c) Firstly we note that the requirement

$$
\alpha<\frac{d}{b(r)}\left(r-\frac{d-1}{d}(n-b(r))\right)
$$

is equivalent to require

$$
\frac{d-1}{d}(n-b(r))+\alpha \frac{b(r)}{d}<r .
$$

Lemma 3 assures the existence of a such $\alpha$. Note that (28) is equivalent to

$$
\underbrace{1+\cdots+1}_{d-1 \text { times }}+(-\beta)<0
$$

which assures that the $\left(d^{+}, n\right)$-strings of $f_{\alpha}$ are only of the type (26). Therefore we have constructed a weight function $f=f_{\alpha}$ with $r$ being nonnegative elements which satisfies (21).

We now concentrate our attention on the subinterval $(((d-1) / d)(n-1),((d-1) / d) n]$. By Proposition 2 there is a unique value of $r$ contained in this subinterval. For this value formula (20) becomes simpler as expressed in the following result.

Corollary 5. Let $r$ be a positive integer such that $r \geq d$ and $((d-1) / d)(n-1)<r \leq((d-1) / d) n$. Then

$$
\gamma(n, d, r) \leq\left(\begin{array}{l}
r \\
d
\end{array}\right)+\left(\begin{array}{c}
r \\
d-1
\end{array}\right)
$$

Proof. The result follows directly from Proposition 4 since $b(r)=1$.

The aim of the next proposition is to try to individuate a lower bound for $\gamma(n, d, r)$ starting from $\phi(f, d)$. Recalling that $\gamma(n, d, r)=\min \left\{\phi(f, d): f \in W_{n}, f^{+}=r\right\}$, we have the following result.

Proposition 6. Let $r$ be a positive integer such that $d \leq r \leq$ $((d-1) / d) n$. Let $f \in W_{n}$, with $f^{+}=r$, as in (3). If

$$
x_{1}+y_{n-r} \geq 0
$$

then

$$
\phi(f, d) \geq\left(\begin{array}{l}
r-1 \\
d-2
\end{array}\right)(n-r)+\left(\begin{array}{l}
r \\
d
\end{array}\right) \geq\left(\begin{array}{l}
r \\
d
\end{array}\right)+\left(\begin{array}{c}
r \\
d-1
\end{array}\right) .
$$

Proof. We can consider the $d$-strings of $\{1, \ldots, n\}$ of type

$$
1 i_{1} \cdots i_{d-2} \mid(n-r)
$$

where $i_{1} \cdots i_{d-2}$ are chosen in $\{2, \ldots, r\}$.

By virtue of $(31)$, each string of the type $(33)$ is a $\left(d^{+}, n\right)$ string of $f$. of type

On the other hand, since $y_{1} \geq y_{2} \geq \cdots \geq y_{n-r}$, each string

$$
1 i_{1} \cdots i_{d-2} \mid k
$$

where $i_{1} \cdots i_{d-2}$ are chosen in $\{2, \ldots, r\}$ and $k$ in $\{1, \ldots, n-r\}$, will be a $\left(d^{+}, n\right)$-string of $f$.

The distinct strings of the type (34) are exactly $\left(\begin{array}{c}r-1 \\ d-2\end{array}\right)(n-$ $r)$. There are moreover all the $\left(d^{+}, n\right)$-strings of $f$ that are the $d$-strings on $\{1, \ldots, r\}$. This proves the first inequality in (32). Moreover, since $r \leq((d-1) / d) n$, we also have $n-r \geq(r /(d-$ 1)). Therefore

$$
\left(\begin{array}{l}
r-1 \\
d-2
\end{array}\right)(n-r) \geq\left(\begin{array}{l}
r-1 \\
d-2
\end{array}\right) \frac{r}{d-1}=\left(\begin{array}{c}
r \\
d-1
\end{array}\right) .
$$

Thus the second inequality also holds.

Proposition 6 leads us to state the following conjecture.

Remark 7. We conjecture that

$$
\gamma(n, d, r)=\left(\begin{array}{l}
r \\
d
\end{array}\right)+\left(\begin{array}{c}
r \\
d-1
\end{array}\right)
$$

where $r \geq d$ and $((d-1) / d)(n-1)<r \leq((d-1) / d) n$.

Note that, in order to prove (36), by Corollary 5 , it is sufficient to show

$$
\gamma(n, d, r) \geq\left(\begin{array}{l}
r \\
d
\end{array}\right)+\left(\begin{array}{c}
r \\
d-1
\end{array}\right) .
$$

In the particular case $n=2 d+2$ and $r=2 d-1$ the previous conjecture has been proved in [3].

In the next result we show that our conjecture (36) is true when $n=d+2$ and $r=d$.

Proposition 8. If $n=d+2$ and $r=d$, then

$$
\gamma(n, d, r)=\left(\begin{array}{l}
r \\
d
\end{array}\right)+\left(\begin{array}{c}
r \\
d-1
\end{array}\right)
$$


Proof. We observe that if $n=d+2$ and $r=d$, then $((d-$ $1) / d)(n-1)<r \leq((d-1) / d) n$. Hence, by Corollary 5 ,

$$
\gamma(n, d, r) \leq\left(\begin{array}{l}
r \\
d
\end{array}\right)+\left(\begin{array}{c}
r \\
d-1
\end{array}\right) .
$$

Therefore, in order to have the thesis, we must prove that

$$
\gamma(n, d, r) \geq\left(\begin{array}{l}
r \\
d
\end{array}\right)+\left(\begin{array}{c}
r \\
d-1
\end{array}\right) .
$$

Let us note that as a direct consequence of Corollary 5 and Proposition 6 it follows that if $r$ is a positive integer with $r \geq d$ such that $((d-1) / d)(n-1)<r \leq((d-1) / d) n$, then

$$
\begin{aligned}
\min & \left\{\phi(f, d): f \in W_{n}, f^{+}=r, x_{1}+y_{n-r} \geq 0\right\} \\
& =\left(\begin{array}{l}
r \\
d
\end{array}\right)+\left(\begin{array}{c}
r \\
d-1
\end{array}\right) .
\end{aligned}
$$

Therefore by (41) inequality (40) is equivalent to the following:

$$
\begin{aligned}
& \min \left\{\phi(f, d): f \in W_{n}, f^{+}=r, x_{k}+y_{n-r}<0\right. \\
& \text { for every } k=1, \ldots, r\} \\
& \geq\left(\begin{array}{l}
r \\
d
\end{array}\right)+\left(\begin{array}{c}
r \\
d-1
\end{array}\right) .
\end{aligned}
$$

So, to prove inequality (40), it is sufficient to prove that, for each $f \in W_{n}$ such that $f^{+}=r$ and $x_{1}+y_{n-r}=x_{1}+y_{2}<0$, we have $\phi(f, d) \geq\left(\begin{array}{l}r \\ d\end{array}\right)+\left(\begin{array}{c}r \\ d-1\end{array}\right)$. We set $p=\left(\begin{array}{c}r \\ d-1\end{array}\right)$ and let $A_{1}, \ldots, A_{p}$ be all the $(d-1)$-strings in $[r]$. At this point we consider the following configurations:

$$
\begin{array}{cc}
A_{1} \mid 1 & i_{1} \mid 2 \\
\vdots & \vdots \\
A_{p} \mid 1 & i_{p} \mid 2,
\end{array}
$$

where $\left\{i_{j}\right\}=[r] \backslash A_{j}(j=1, \ldots, p)$. Since $f$ is a weight function and $x_{i_{j}}+y_{2}<0$ for $j=1, \ldots, p$, it follows that all the $d$-strings $A_{1}\left|1, \ldots, A_{p}\right| 1$ are nonnegative $d$-tuple of the form $(d-1,1)$ associated with $f$. Therefore $\phi(f, d) \geq$ $\left(\begin{array}{l}r \\ d\end{array}\right)+p=\left(\begin{array}{l}r \\ d\end{array}\right)+\left(\begin{array}{c}r \\ d-1\end{array}\right)$. This proves (42), and hence also (40) is proved.

In Table 1 we list all the known values of $\gamma(n, d, r)$ in the range $((d-1) / d)(n-1)<r \leq((d-1) / d) n$. Let us note that when the integer $d$ is fixed and $n$ runs in $\{d+1, d+2, \ldots\}$, by Proposition 2, we find at most an integer value $r$ such that $((d-1) / d)(n-1)<r \leq((d-1) / d) n$.

In Table $1, k=1,2, \ldots$ and $t \in\{1, \ldots, d\}$. Moreover, if $t=1$, then there does not exist an integer $r$ such that $((d-1) / d)(n-1)<r \leq((d-1) / d) n$. In the last column we have marked the cases in which the conjecture (36) is proved. In particular, we note that, for every $k$, the case $t=d$ corresponds to $n=(k+1) d$, which implies $r=(k+1)(d-1)=$ $((d-1) / d) n$. For these values of $r$ we know that (36) holds (see [3]) and the conjecture (36) is true.
TABLE 1

\begin{tabular}{lccl}
\hline$n$ & $r$ & $n-r$ & \\
\hline$d+1$ & $\nexists$ & $\nexists$ & \\
$d+2$ & $d$ & 2 & Proposition 8 \\
$\vdots$ & $\vdots$ & $\vdots$ & \\
$d+(d-1)$ & $2 d-3$ & 2 & \\
$2 d$ & $2 d-2$ & 2 & Proposition 2.5 in [3] \\
\hline $2 d+1$ & $\nexists$ & $\nexists$ & \\
$2 d+2$ & $2 d-1$ & 3 & Proposition 2.4 in [3] \\
$\vdots$ & $\vdots$ & $\vdots$ & \\
\hline$k d+1$ & $\nexists$ & $\nexists$ & \\
$\vdots$ & $\vdots$ & $\vdots$ & \\
$k d+t$ & $k(d-1)+(t-1)$ & $k+1$ & \\
$\vdots$ & $\vdots$ & $\vdots$ & \\
$(k+1) d$ & $(k+1)(d-1)$ & $(k+1)$ & Proposition 2.5 in [3] \\
$\vdots$ & $\vdots$ & $\vdots$ &
\end{tabular}

We give now a simple combinatorial interpretation of the inequalities

$$
\frac{d-1}{d}(n-1)<r \leq \frac{d-1}{d} n
$$

For this purpose let us note that the last inequalities are equivalent to the following:

$$
(n-r-1)(d-1)<r \leq(n-r)(d-1) .
$$

Let now $r$ be a positive integer that satisfies (45) and $f \in W_{n}$, with $f^{+}=r$, as in (3). Let us consider the following representation:

$$
\begin{gathered}
\llcorner\lrcorner+\llcorner\lrcorner+\cdots+\llcorner\lrcorner+k_{1} \\
\llcorner\lrcorner+_{\llcorner\lrcorner}+\cdots+\llcorner\lrcorner+k_{2} \\
\vdots \\
\llcorner\lrcorner+_{\llcorner\lrcorner}+\cdots+\llcorner\lrcorner+k_{n-r-1} \\
\llcorner\lrcorner+_{\llcorner\lrcorner}+\cdots+{ }_{\llcorner\lrcorner}+k_{n-r},
\end{gathered}
$$

where every \llcorner\lrcorner can be seen as a "box" initially empty and every row contains $d-1$ boxes. Each of such boxes can be occupied by at most one nonnegative element of $f$. Thus (45) is equivalent to state that $n-r-1$ rows in (46) must be completely occupied, whereas the last row must contain at least a nonempty box and, furthermore, the number of nonnegative elements of $f$ cannot exceed the number of empty boxes in (46). This combinatorial interpretation of (45) suggests to examine firstly the $\left(d^{+}, n\right)$-strings of $f$ of the form $+\cdots+-$, that is, a subset with $d-1$ nonnegative elements and only one negative. 


\section{Conclusions and Further Developments}

In this paper we continue the research approach started in $[1,3]$ to the problem of determining new identities and new bounds concerning the numbers $\gamma(n, d, r)$. After a brief introduction to these numbers and their combinatorial context, we establish some preliminary results necessary to delimit the range of the integers parameters $n, d$, and $r$ that we study. Next we give a relevant upper bound for numbers $\gamma(n, d, r)$ (Proposition 4). Afterwards we focus our attention on the range $((d-1) / d)(n-1)<r \leq((d-1) / d) n$ (Corollary 5$)$. In this context, Proposition 6 and the study of $\phi(f, d)$ bring us to conjecture a strong lower bound for $\gamma(n, d, r)$ on the subinterval $((d-1) / d)(n-1)<r \leq((d-1) / d) n$. Finally, we prove our conjecture in the case $n=d+2$ and $r=d$, and this provides an exact value for $\gamma(r+2, r, r)$. We think that our approach of dividing the intervals of variation of the integers parameters $n, d$, and $r$ in particular types of subintervals can be useful in order to determine further upper and lower bound for $\gamma(n, d, r)$. In future papers our purpose will be:

(i) to study other subintervals, trying to extend and generalize the validity of the results of this paper;

(ii) to prove conjecture $(36)$ in all the range $((d-1) / d)(n-$ $1)<r \leq((d-1) / d) n$.

\section{Conflict of Interests}

The author declares that there is no conflict of interests regarding the publication of this paper.

\section{Acknowledgment}

The author is extremely thankful to one unknown referee for his useful comments and suggestions that have helped in improving the readability of this paper.

\section{References}

[1] G. Chiaselotti, "On a problem concerning the weight functions," European Journal of Combinatorics, vol. 23, no. 1, pp. 15-22, 2002.

[2] C. Bisi and G. Chiaselotti, "A class of lattices and boolean functions related to the Manickam-Miklos-Singhi Conjecture," Advances in Geometry, vol. 13, no. 1, pp. 1-27, 2013.

[3] G. Chiaselotti, G. Infante, and G. Marino, "New results related to a conjecture of Manickam and Singhi," European Journal of Combinatorics, vol. 29, no. 2, pp. 361-368, 2008.

[4] G. Marino and G. Chiaselotti, "A method to count the positive 3 -subsets in a set of real numbers with non-negative sum," European Journal of Combinatorics, vol. 23, no. 5, pp. 619-629, 2002.

[5] G. Chiaselotti, G. Marino, and C. Nardi, "A minimum problem for finite sets of real numbers with nonnegative sum," Journal of Applied Mathematics, vol. 2012, Article ID 847958, 15 pages, 2012.

[6] C. Bisi, G. Chiaselotti, and P. A. Oliverio, "Sand piles models of signed partitions with $d$ piles," ISRN Combinatorics, vol. 2013, Article ID 615703, 7 pages, 2013.
[7] G. Chiaselotti, T. Gentile, G. Marino, and P. A. Oliverio, "Parallel rank of two sandpile models of signed integer partitions," Journal of Applied Mathematics, vol. 2013, Article ID 292143, 12 pages, 2013.

[8] J. A. Aledo, S. Martínez, F. L. Pelayo, and J. C. Valverde, "Parallel discrete dynamical systems on maxterm and minterm Boolean functions," Mathematical and Computer Modelling, vol. 55, no. 3-4, pp. 666-671, 2012.

[9] J. A. Aledo, S. Martínez, and J. C. Valverde, "Parallel dynamical systems over directed dependency graphs," Applied Mathematics and Computation, vol. 219, no. 3, pp. 1114-1119, 2012.

[10] J. A. Aledo, S. Martínez, and J. C. Valverde, "Parallel discrete dynamical systems on independent local functions," Journal of Computational and Applied Mathematics, vol. 237, no. 1, pp. 335339.

[11] J. L. G. Guirao, F. L. Pelayo, and J. C. Valverde, "Modeling the dynamics of concurrent computing systems," Computers and Mathematics with Applications, vol. 61, no. 5, pp. 1402-1406, 2011.

[12] F. L. Pelayo and J. C. Valverde, "Notes on 'Modeling the dynamics of concurrent computing systems"' Computers and Mathematics with Applications, vol. 64, no. 4, pp. 661-663, 2012. 


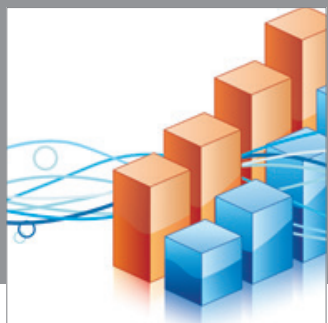

Advances in

Operations Research

mansans

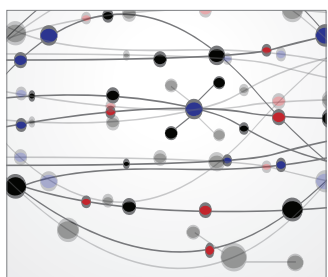

The Scientific World Journal
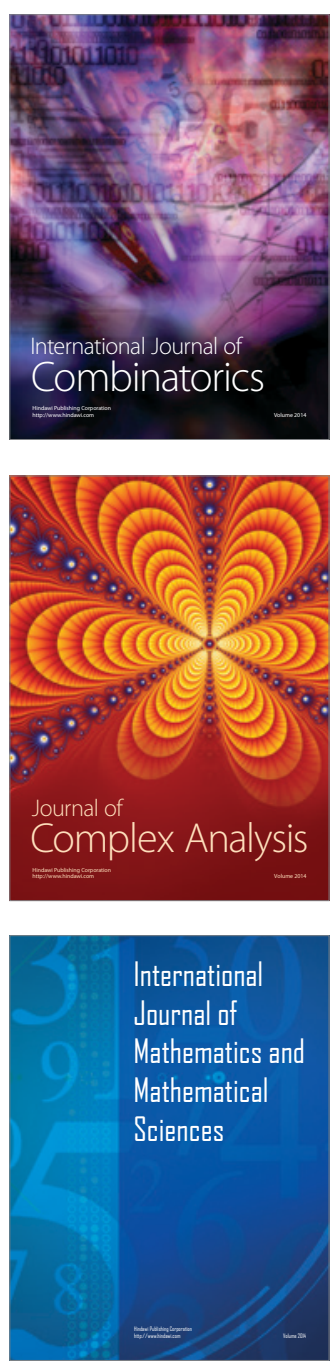
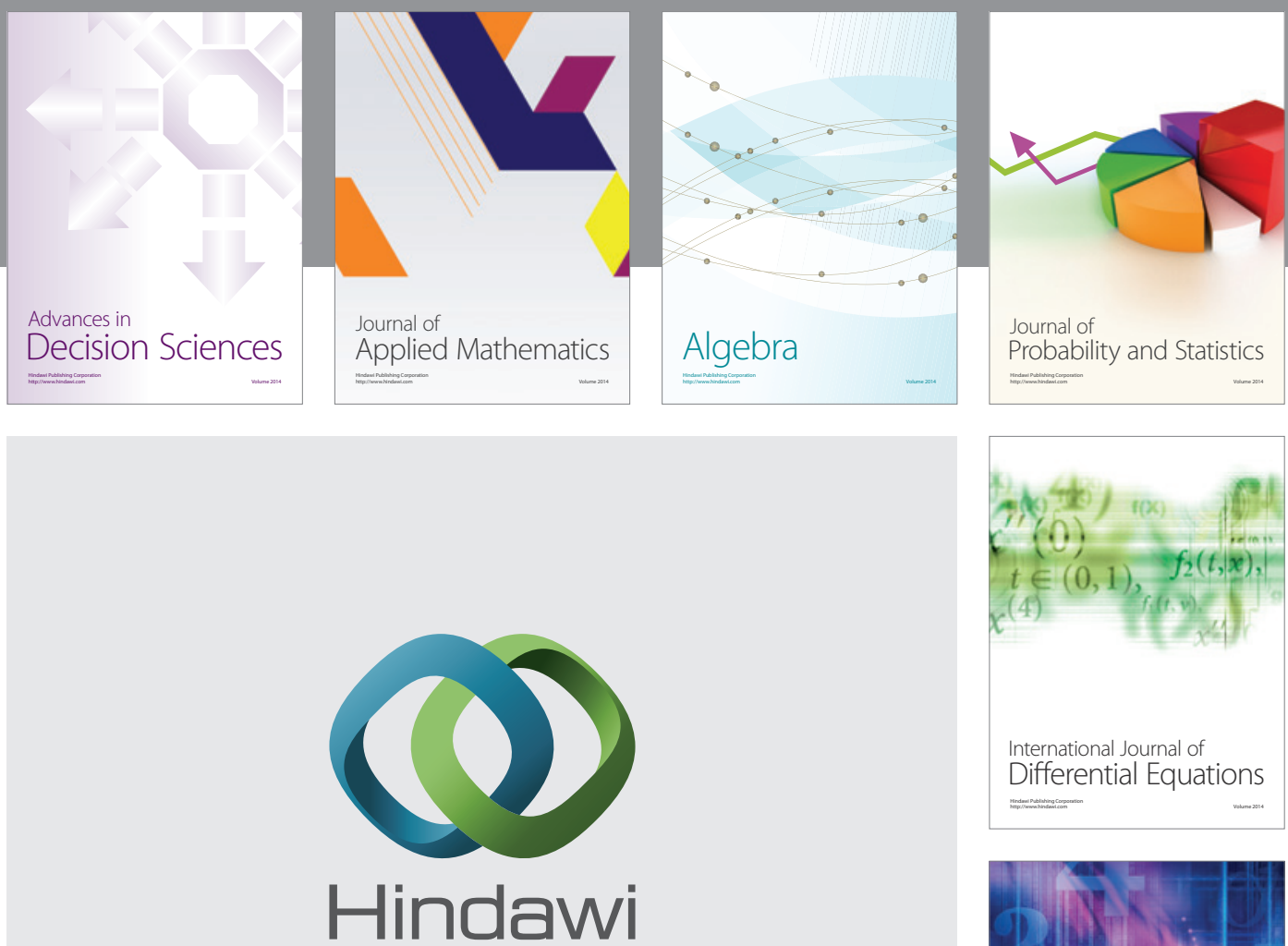

Submit your manuscripts at http://www.hindawi.com
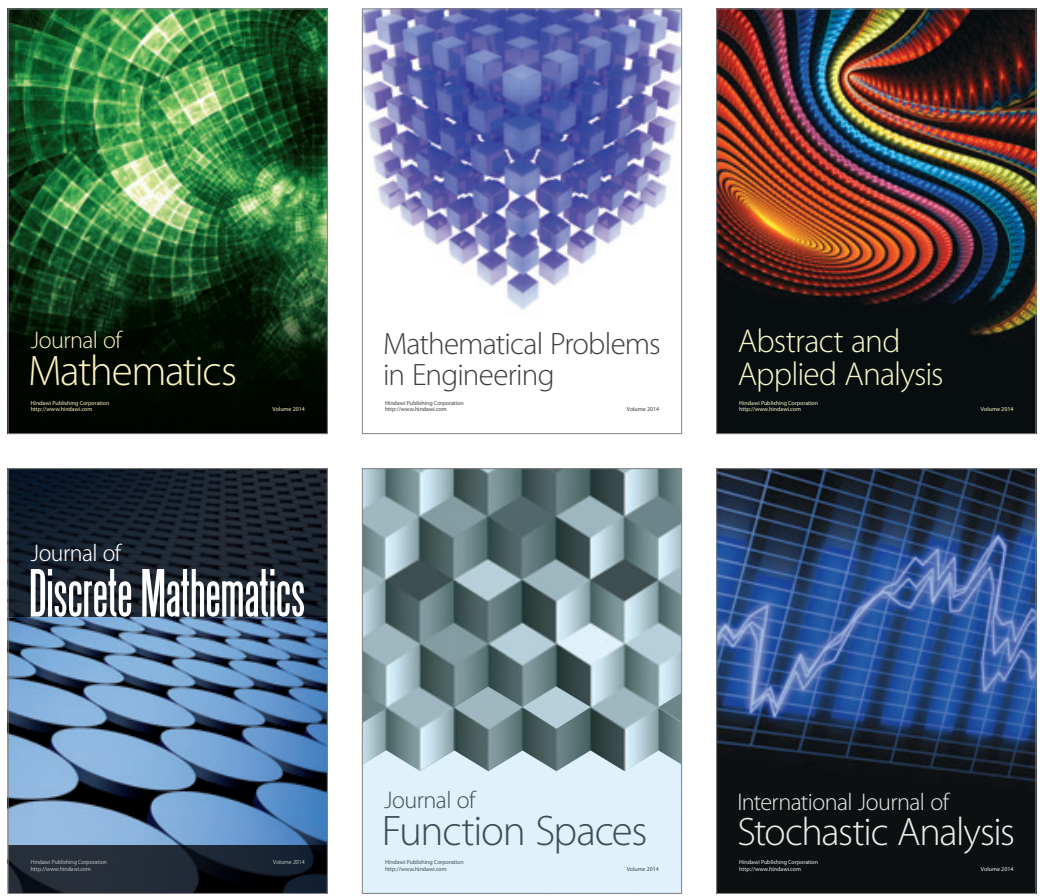

Journal of

Function Spaces

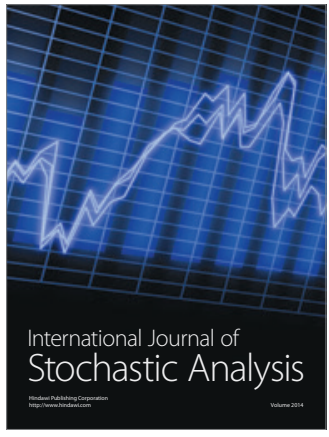

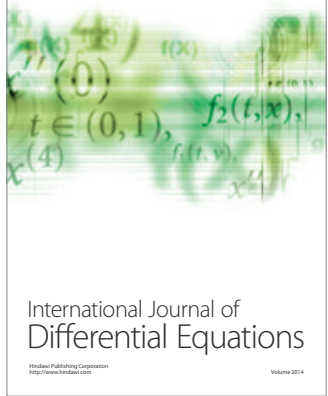
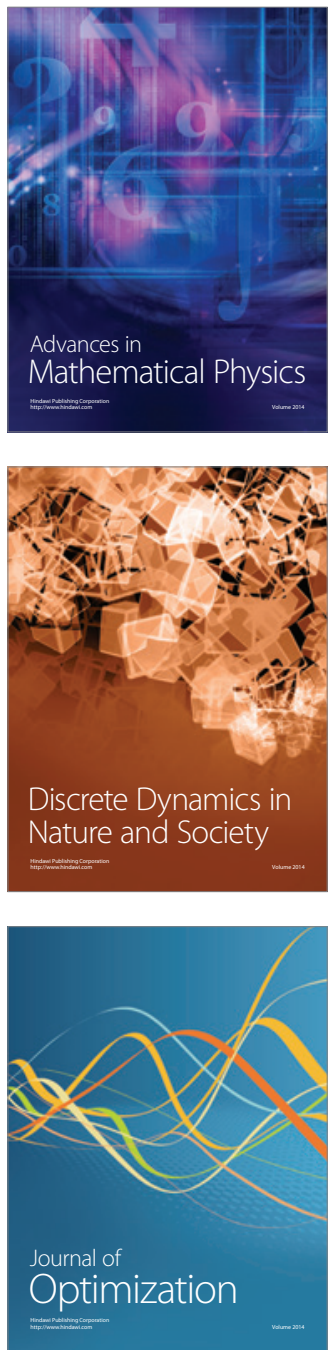